\title{
Mélange à quatre ondes intracavité et fonctions logiques optiques
}

\author{
B. Thedrez $\left({ }^{*}\right)$ et R. Frey $\left({ }^{+}\right)$ \\ (*) Groupe Optique des Matériaux, Ecole Nationale Supérieure des Télécommunications, 46, rue Barrault, \\ 75634 Paris Cedex 13, France \\ $\left({ }^{+}\right)$Laboratoire d'Optique Quantique, Centre National de la Recherche Scientifique, Ecole Polytechnique, \\ 91128 Palaiseau Cedex, et Groupe Optique des Matériaux, Ecole Nationale Supérieure des Télécommunica- \\ tions, 46, rue Barrault, 75634 Paris Cedex 13, France
}

(Reçu le 15 décembre 1986, accepté le 5 février 1987)

\begin{abstract}
Résumé. - Une nouvelle technique utilisant le mélange intracavité de quatre ondes quasi dégénérées en fréquence et l'injection optique permet la réalisation de portes logiques optiques. Cette technique est fondée sur le filtrage de fréquence d'ondes interagissant de façon non linéaire dans des diodes laser à semiconducteur. Après un exposé du principe, le cas des portes OU, INV et ET est spécifiquement étudié. Avantages et inconvénients de la technique sont également discutés.
\end{abstract}

\begin{abstract}
A new technique using intracavity nearly degenerate four-wave mixing and injection-locking processes permits the realization of optical logic gates. This technique is based on frequency filtering of waves interacting nonlinearly in semiconductor laser diodes. The principle of this technique is described, with OR, INV, and AND gates being specifically studied. Advantages and disadvantages of this technique are also discussed.
\end{abstract}

\section{Introduction.}

L'utilisation de la bistabilité optique obtenue dans des étalons de Fabry-Pérot non linéaires permet la réalisation de portes logiques toute optique [1]. La porte NOR peut quant à elle être obtenue sans étalon Fabry-Pérot en utilisant l'effet d'écrantage excitonique dans les semiconducteurs [2]. Toutefois, se pose alors le problème de changement de fréquence opéré lors du fonctionnement de la porte, ce qui hypothèque largement la mise en série de tels dispositifs. D'autre part, les pertes inhérentes à l'utilisation de dispositifs non linéaires absorbants (bistables ou inverseurs) sont également un facteur limitatif à la mise en cascade de dispositifs passifs. La réalisation des interactions non linéaires dans des milieux amplificateurs tels que les lasers ne présente pas cet inconvénient, puisque les signaux de sortie sont alors généralement plus intenses que les signaux d'entrée. En raison de leur faible encombrement les lasers à semiconducteur ont été particulièrement étudiés : des fonctions logiques optiques ont effectivement été réalisées dans des configurations hybrides utilisant à la fois des éléments optiques et électroniques [3, 4].
Dans cet article nous présentons une nouvelle technique permettant la réalisation de portes optiques dans des diodes laser à semiconducteur. Cette technique toute optique est fondée sur l'utilisation du mélange intracavité de quatre ondes quasi dégénérées en fréquence $[5,6]$ et celle de l'injection optique [7]. Ces interactions sont réalisées dans des diodes lasers opérant en dessous ou au-dessus du seuil d'auto-oscillation. De ce fait, les signaux de sortie sont toujours largement amplifiés. Cette nouvelle technique diffère des procédés couramment utilisés en ce sens que l'obtention du signal de sortie résulte d'un changement de fréquence et non pas d'un seuillage en intensité.

Dans le deuxième paragraphe nous présentons le principe de fonctionnement du mélange à quatre ondes et de l'injection optique. Le troisième paragraphe décrit le dispositif expérimental utilisé pour mettre en évidence le fonctionnement de ces portes optiques. Dans le quatrième paragraphe nous détaillons le mode opérationnel des portes OU, INV et ET et nous donnons les principaux résultats expérimentaux. Enfin, la conclusion est l'occasion de discuter les avantages et les inconvénients de ces dispositifs. 
2. Mélange à quatre ondes intracavité et injection optique.

Le processus de mélange intracavité de quatre ondes quasi dégénérées en fréquence est réalisé dans une diode laser à semi-conducteur opérant en dessous ou au-dessus du seuil d'oscillation laser. Pour ce processus schématisé sur la figure 1 , le milieu non linéaire est constitué du laser à semiconducteur lui-même.

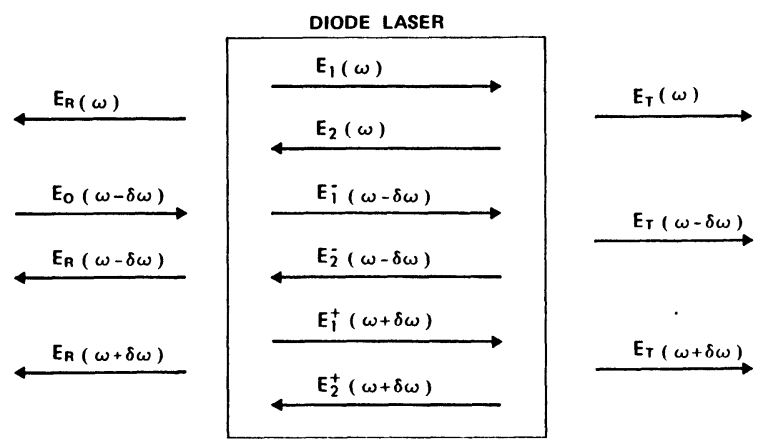

Fig. 1. - Géométrie du mélange intracavité de quatre ondes quasi dégénérées en fréquence.

[Geometry of intracavity nearly-degenerate four-wave mixing.]

Les ondes de pompe $E_{1}(\omega)$ et $E_{2}(\omega)$ sont dues soit à l'effet laser dans la diode (fonctionnement au-dessus du seuil), soit à l'amplification intracavité d'un signal de pompe externe $E_{0}(\omega)$ (fonctionnement au-dessous du seuil). Quand l'onde $E_{0}^{-}(\omega-\delta \omega)$ de fréquence sonde $\omega-\delta \omega$ est injectée dans le laser selon l'axe de la cavité, les ondes conjuguées $E_{1}^{+}(\omega+\delta \omega)$ et $E_{2}^{+}(\omega+\delta \omega)$ sont générées par interaction paramétrique des ondes de pompe $E_{1}(\omega)$ et $E_{2}(\omega)$ d'une part, et sonde $E_{1}^{-}(\omega-\delta \omega)$ et $E_{2}^{-}(\omega-\delta \omega)$ d'autre part [8]. De cette manière, on dénombre des faisceaux réfléchis et transmis d'amplitude et de fréquence $E_{\mathrm{R}, \mathrm{T}}(\omega i)$ avec $\omega i=\omega$, $\omega-\delta \omega$, et $\omega+\delta \omega$ pour respectivement les ondes pompes, sondes et conjuguées. Il est, par conséquent, possible d'observer des signaux à chacune de ces trois fréquences si l'on dispose d'un filtre optique, étalon de Fabry-Pérot, interféromètre à fibre optique ou coupleur directionnel par exemple.

Dans le cas de l'injection optique le laser à semiconducteur opère au-dessus du seuil ; il génère donc lui-même les ondes réfléchies et transmises à la fréquence pompe $\omega$. L'injection d'un faisceau sonde est également réalisée à la fréquence $\omega-\delta \omega$, mais dans ce cas l'amplitude $E_{0}^{-}(\omega-\delta \omega)$ de cette sonde injectée est considérablement plus importante que dans le cas du mélange à quatre ondes. En conséquence de la forte intensité de l'onde sonde, l'oscillation est arrêtée à la fréquence pompe $\omega$ et il se produit une amplification très importante à la fré- quence sonde $\omega-\delta \omega$ qui est alors la seule fréquence délivrée par le dispositif.

\section{Dispositif expérimental.}

Les expériences ont été réalisées en utilisant des diodes laser à semiconducteur GaAlAs comme milieu non linéaire mais aussi comme sources lumineuses. Le dispositif expérimental présenté dans la figure 2 correspond au cas où l'interaction non linéaire est réalisée dans la diode laser LD2 opérant au-dessus du seuil. Le laser génère donc les ondes de pompe à la fréquence $\omega$. Le faisceau sonde incident de fréquence $\omega-\delta \omega$ provenant de la diode laser LD1 est injecté dans LD2 selon l'axe de la cavité de ce laser. Quand l'intensité du faisceau sonde injecté est faible le mélange à quatre ondes intracavité réalisé dans LD2 génère le faisceau conjugué de fréquence $\omega+\delta \omega$. Les trois faisceaux pompe, sonde et conjugué sont observés en transmission (en fait ils pourraient également être observés en réflexion).

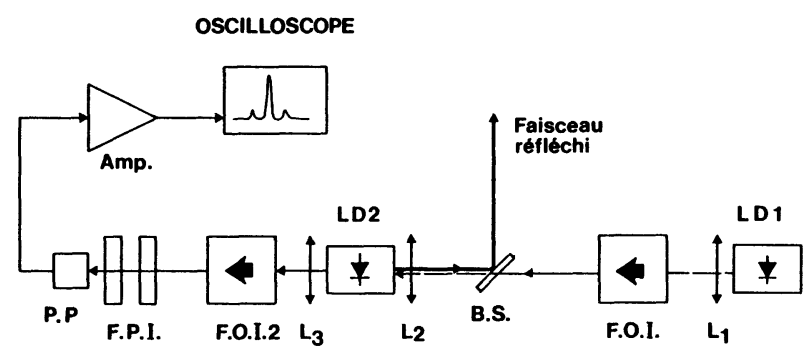

Fig. 2. - Dispositif expérimental servant à la mise en évidence du fonctionnement des portes logiques. LD1LD2 = diodes lasers ; FOI1-FOI2 = isolateurs optiques ; L1-L3 = lentilles ; FPI = interféromètre de Fabry-Pérot ; $\mathrm{PP}=\mathrm{PIN} ; \mathrm{BS}=$ Séparateur de faisceau.

[Experimental set-up used to demonstrate the operation of logical gates. LD1-LD2 = Laser Diodes ; FOI1-FOI2 = Faraday Isolators ; L1-L3 = Lenses ; FPI = Fabry-Pérot Interferometer ; PP = PIN Photodiode ; BS = Beam Splitter.]

Les isolateurs optiques à effet Faraday FOI1 et FOI2 sont utilisés pour éviter tout couplage parasite entre les deux lasers ou avec l'optique de mesure. Les différents faisceaux sont séparés en fréquence grâce à un interféromètre de Fabry-Pérot d'épaisseur variable et observés en aval par une photodiode PIN connectée à un oscilloscope. Si l'intensité sonde donnée par le laser LD1 est élevée, il n'y a plus d'onde conjuguée car le laser est verrouillé à la fréquence sonde $\omega-\delta \omega$. Néanmoins, l'observation est toujours réalisée au travers de l'interféromètre FPI.

Quand la diode laser LD2 servant de milieu non linéaire opère en dessous du seuil laser, l'injection - à la fréquence pompe $\omega$ - est réalisée grâce à une 
troisième diode laser et un séparateur de faisceau placé entre LD1 et FOI1.

Enfin pour obtenir le fonctionnement des portes logiques les faisceaux injectés sont choppés mécaniquement, l'observation des signaux d'entrée étant réalisée par l'intermédiaire de photodiodes PIN.

\section{Principales opérations logiques optiques.}

Les trois principales opérations logiques : OU, ET et INV peuvent aisément être réalisées selon les principes décrits dans le paragraphe 2 .

\section{Porte $O U$}

Le principe de fonctionnement de la porte OU est présenté dans la figure 3 . Une telle porte utilise deux lasers émettant en régime continu à la fréquence pompe $\omega$. Deux signaux choppés de faible intensité $A$ et $B$ sont chacun injectés dans un laser à la fréquence $\omega-\delta \omega$. Les deux filtres de fréquence représentés sur la figure transmettent uniquement la fréquence conjuguée $\omega$. De cette manière, si un signal est envoyé à la fréquence sonde en $\mathrm{A}$, ou en $B$, ou même dans les deux canaux d'entrée, un signal de sortie $R$ est obtenu à la fréquence $\omega+\delta \omega$. Au contraire, aucun signal ne peut être observé à cette fréquence conjuguée s'il n'y a d'entrée ni en A ni en B.

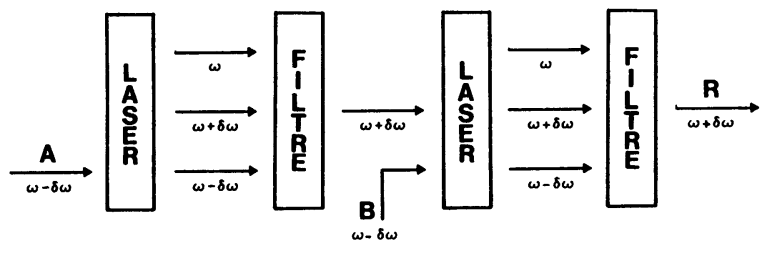

Fig. 3. - Schéma de principe de la porte OU.

[Schematic arrangement of the OR gate.]

\section{Porte INV}

La figure 4 montre une réalisation de porte logique inverseuse utilisant l'injection optique. En effet, si le laser oscillant à la fréquence $\omega$ est injecté par un signal A d'intensité suffisante, le verrouillage du laser s'effectue à cette fréquence sonde qui est par conséquent la seule qui soit observable à la sortie du laser. Si le filtre optique ne transmet que la fréquence pompe $\omega$ le signal $R$ n'est obtenu qu'en absence du signal sonde, réalisant ainsi la porte logique INV.

Le fonctionnement de cette porte INV est démontré expérimentalement sur les traces d'oscilloscope présentées dans la figure 5. Les deux figures (a) et (b) correspondent au fonctionnement du dispositif en répéteur ou en inverseur. Dans les deux cas la trace d'oscilloscope inférieure montre le signal d'entrée $\mathrm{A}$ à la fréquence $\omega-\delta \omega$. Dans le cas du répéteur (Fig. 5a) le signal transmis par l'interféromètre de Fabry Pérot est à la même fréquence sonde

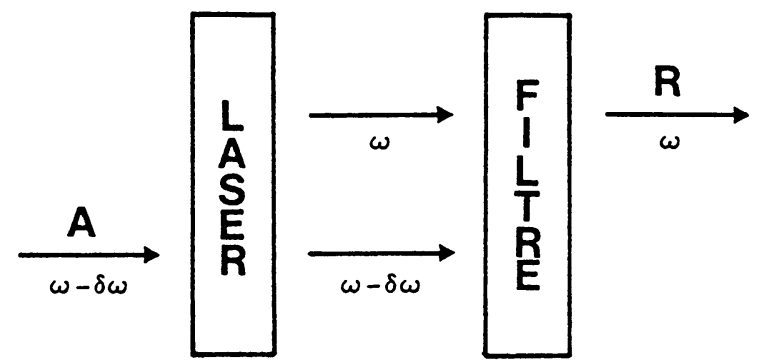

Fig. 4. - Schéma de principe de la porte INV.

[Schematic arrangement of the INV gate.]

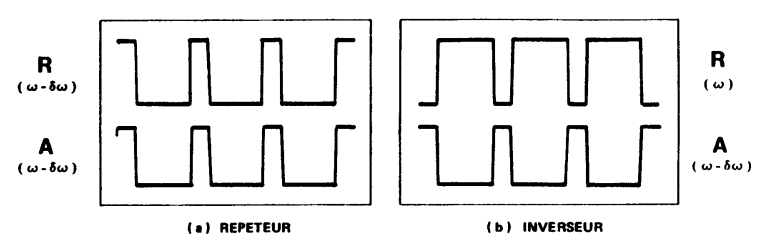

Fig. 5. - Démonstration expérimentale du fonctionnement de la porte INV.

[Experimental evidence of INV gate operation.]

que le signal d'entrée. De ce fait on observe seulement une très forte amplification du signal sonde incident. Par contre, quand le signal filtré est à la fréquence $\omega$, l'inversion se produit en même temps que l'amplification (Fig. 5b).

Porte ET

Le mélange intracavité de quatre ondes quasi dégénérées en fréquence réalisé dans des diodes laser opérant légèrement en dessous du seuil laser permet de réaliser très simplement une porte ET (Fig. 6). Cette porte est composée d'un amplificateur laser et d'un filtre optique. Des signaux d'entrée A et $B$ de fréquences respectives $\omega$ et $\omega-\delta \omega$ sont injectés dans l'amplificateur laser. Le filtre optique rejette deux fréquences et transmet la fréquence conjuguée $\omega+\delta \omega$, de telle sorte que le signal de sortie $R$ n'est obtenu qu'en la présence simultanée des signaux $A$ et $B$.

Les résultats expérimentaux obtenus lors du fonctionnement de la porte ET sont présentés sur les

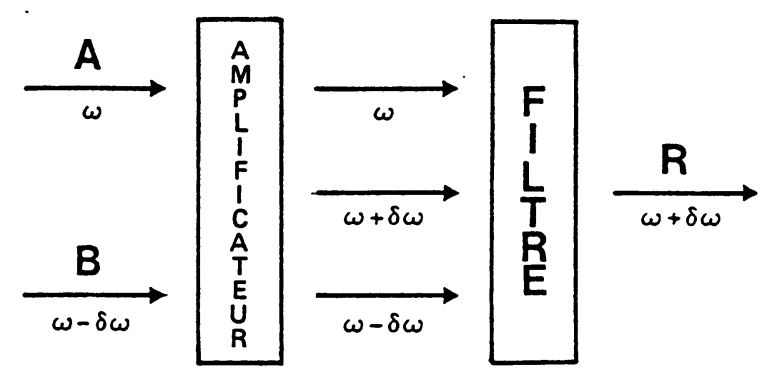

Fig. 6. - Schéma de principe de la porte ET.

[Schematic arrangement of the AND gate.] 
traces d'oscilloscopes de la figure 7. Les deux traces inférieures correspondent aux entrées A et B et, comme prévu, le signal $\mathbf{R}$ de la trace supérieure ne présente une impulsion qu'en la présence simultanée de $A$ et $B$.

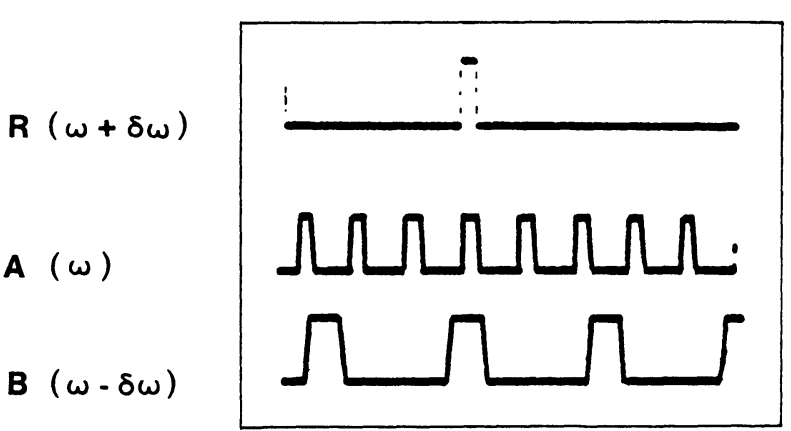

Fig. 7. - Démonstration expérimentale du fonctionnement de la porte ET.

[Experimental evidence of AND gate operation.]

Toutes ces expériences ont été réalisées avec des diodes laser GaAlAs émettant à $0,85 \mu \mathrm{m}$. Bien évidemment, rien ne s'oppose dans le principe à un fonctionnement à 1,3 ou $1,5 \mu \mathrm{m}$ mieux adapté aux applications télécommunications. Les écarts de fréquence entre les différents lasers sont de l'ordre de quelques gigahertz, autorisant un temps de réponse subnanoseconde compatible avec le temps de recombinaison des paires électron-trou par émission stimulée. De plus, avec cette technique, la cadence de commutation est en principe uniquement limitée par le temps de réponse du dispositif. Enfin, pour obtenir un fonctionnement monomode, les lasers utilisés émettent une puissance de quelques milliwatts. Toutefois, une telle puissance n'est pas dans le principe nécessaire au bon fonctionnement de la technique et les puissances réelles injectées sont bien inférieures (quelques dizaines de microwatts).

\section{Conclusion.}

Dans cet article, nous avons proposé et démontré le fonctionnement d'un nouveau type de portes logiques. Leur principe opérationnel est fondé sur le filtrage de fréquence de différentes ondes interagissant de façon non linéaire dans des diodes laser à semiconducteur. Cette technique est particulièrement bien adaptée à la mise en cascade des différents dispositifs. En effet, étant réalisée dans des milieux fortement amplificateurs, le problème du niveau de signal d'entrée ne se pose plus. De plus étant donné que les interactions non linéaires ne sont pas sensibles au signe du décalage de fréquence, il est possible de réaliser des systèmes en cascade avec seulement trois types de dispositifs opérant aux fréquences $\omega, \omega-\delta \omega$ et $\omega+\delta \omega$. L'inconvénient essentiel de ce nouveau type de fonctions logiques réside en la difficulté actuelle d'intégrer massivement de tels dispositifs. En effet, il est nécessaire de stabiliser la température des diodes lasers car cet élément influence considérablement le paramètre essentiel de l'interaction à savoir la fréquence d'émission du laser lui-même. Ce problème est à l'heure actuelle résolu à l'échelle du laboratoire avec un dispositif de faible dimension mais évidemment non intégrable. Cet inconvénient devient encore plus important si l'on s'attache à l'obtention d'un traitement massivement parallèle de l'information. Toutefois, les importantes recherches technologiques réalisées actuellement sur les lasers à semiconducteur bloqués en phase pourraient servir de base au développement de réseaux de lasers indépendants qui permettraient alors une intégration importante.

Les gros problèmes technologiques posés par l'intégration de tels dispositifs ne semblant pas complètement insurmontables, il paraît important de mieux caractériser ces portes logiques. Les recherches sont donc poursuivies dans cette voie en particulier en vue de déterminer le temps de réponse de ces dispositifs et de réaliser une optimisation énergétique de leur fonctionnement.

\section{Bibliographie}

[1] Peyghambarian, N., Gibbs, H. M., Hulin, D., ANTONETTI, A., Migus, A. and MYSYROWICZ, A., Proc. of High Speed Electronics, Stockholm (Suède), 1986 (à paraître chez Springer-Verlag).

[2] Collet, J. et Amand, T., Revue Phys. Appl. Ce volume.

[3] Tsang, W. T., Olson, N. A. and Logan, R. A., IEEE J. Quantum Electron. QE-19 (1983) 1621.

[4] OKumura, K., OGAWA, Y., ITO, H. and INABA, H., Opt. Lett. 9 (1984) 519.
[5] Nakajima, H. and Frey, R., Phys. Rev. Lett. 54 (1985) 1798.

[6] Nakajima, H. and Frey, R., Appl. Phys. Lett. 47 (1985) 769.

[7] Goldberg, L., TAYlor, H. F. and Weller, J. F., IEEE J. Quantum. Electron. QE-20 (1984) 1226.

[8] NAKAJIMA, H. and FreY, R., IEEE J. Quantum Electron. QE-22 (1986) 1349. 